\title{
Les Effets de la Violence Chez L'Enfant et ses Tentatives de Traitement dans L'Apres Coup
}

\author{
Blanquet Brigitte \\ L'université Lumière Lyon
}

\begin{abstract}
RESUMÉ - A travers cet article, l'auteur explique que les effets de la violence sur l'enfant ne s'observent bien souvent, que dans l'après coup et notamment à l'adolescence. L'adolescence constitue une opportunité qui permet que se reprennent et se traitent les nombreux traumatismes qui ont marqué la psyché de l'enfant. Grâce au processus de répétition-création, l'adolescent remet en scène les traces d'anciens vécus traumatiques non assimilés pour essayer de les traiter. Pour démontrer ce postulat, l'auteur s'appuie sur une situation clinique, extraite de deux ans de prise en charge psychothérapique. Cet exemple permet ainsi, de rendre compte de ce phénomène de reprise, de mise en scène et des effets du travail psychique après coup.
\end{abstract}

Mots-cléfs: Violence; souffrance psychique; adolescence; travail du traumatique; processus de répétition-création; psychothérapie.

\section{The Effects of Violence against Children and Treatment Attempts Years Afterwards}

\begin{abstract}
In this article the author argues that the effects of violence against children are often noticed only years later, especially during adolescence. Adolescence is an opportunity that allows to regain and treat the many injuries that have affected the psyche of the child. By means of the process of repetition-creation, the teenager can act out traces of old traumatic experiences which were not assimilated and try to treat them. To demonstrate this assumption, the author uses a clinical situation, extracted from two years of psychotherapeutic treatement. This example makes it possible to account for the phenomenon of recovery, acting out and the effects of psychotherapeutic treatment years afterwards the traumatic experience.
\end{abstract}

Keywords: Violence, mental suffering; adolescence; traumatic experiences; process of repetition-creation; psychotherapy.

\section{O Efeito da Violência contra Crianças e o Tratamento na Adolescência}

\begin{abstract}
RESUMO - Neste artigo a autora argumenta que os efeitos da violência contras as crianças são, muitas vezes, percebidos apenas anos mais tarde, especialmente durante a adolescência. Nesta fase é possível recuperar e tratar os efeitos provocados pela violência na psique da criança. Por meio do processo de criação-repetição o adolescente pode agir sobre os vestígios de antigas experiências traumáticas que não foram assimilados e tentar tratá-los. Para demonstrar esta hipótese a autora utiliza uma situação clínica, extraída de dois anos de tratamento psicoterapêutico. Este exemplo torna possível explicar o fenômeno de recuperação agindo e os efeitos do tratamento psicoterápico anos depois da experiência traumática.
\end{abstract}

Palavras-Chave: Violência; Sofrimento mental; Adolescência; Experiências traumáticas; Processo de repetição-criação; Psicoterapia.

Les effets de la violence subie et vécue par l'enfant sont difficilement mesurable in situ et nécessite de les penser dans un complexe développemental dynamique. Auparavant un pré requis s'avère indispensable pour observer la nature de ces effets. Ce pré-requis invite à la prise de distance nécessaire face à l'attraction exhibitionniste ou voyeuriste que génèrent les scènes de violences agies ou subies, a fortiori quand il s'agit d'enfant.

A travers cet article, nous apporterons notre contribution afin de soutenir l'apport éclairant de la psychologie

1 Endereço para correspondência: Le village, 26300. Barbière, France. Fone: +33 04454745 95. Email: brig.blanquet@laposte.net. clinique au regard de ces problématiques. Notre position est résoluement clinique, d'une part pour ne pas perdre le sujet face à l'excès d'excitation que toutes formes de violences suscitent, et d'autre part pour accompagner la façon dont il tente dans l'après coup de se déprendre des ces effets. Ainsi, nous rendrons compte de la manière dont un enfant exposé à des scènes de violence, essaie de les traiter dans l'après coup. Les concepts de traumatisme, et d'après coup Freud (1896) constitueront deux levier à partir desquel nous mettrons en lumière l'importance du travail du traumatique au regard des processus de symbolisation. 


\section{Du point de vue développemental}

Traiter des effets de la violence du point de vue du traumatisme, suppose en préambule de se situer du côté de l'enfant, c'est à dire de la manière dont chaque enfant vit, perçoit, interprète et tente de s'approprier la nature de l'expérience à laquelle il est confronté. En effet, l'enfant essaie de signifier ce qui lui arrive en fonction de sa capacité à comprendre, au regard des liens qu'il entretient avec les adultes et du fait de son immaturité psychique. Le monde relationnel de l'adulte est dissemblable de celui de l'enfant. Ces deux mondes font appel à deux réalités psychiques différentes, constituées de logiques et des lois différentes pouvant parfois même, s'entrechoquer. Pour préciser ces différences, Férenczi (1932) parle de confusion de langue entre l'enfant et l'adulte. C'est pourquoi le rapport de chaque enfant à une scène de violence reste singulier et inhérent à la manière dont elle est vécue et interprétée par lui. Les effets qui ont un impact traumatique sur sa psyché, s'observent bien souvent dans l'après coup, c'est à dire dans une reprise ultérieure de ce que l'enfant n'a pu traiter en tant qu'effets délétères auquel son environnement l'a exposé. Pour avancer dans ce premier constat, nous proposons de considérer que ce qui a marqué la psyché de l'enfant, fait retour dans une autre temporalité. Autrement dit, ce qu'il n'a pu traiter revient et se remet en scène à l'adolescence. L'enfant devenu adolescent rejoue ce à quoi il a été confronté et qui a été inintégrable pour lui. Il s'agit pour lui d'une potentialité autre qui va le conduire à « se contruire un passé » Aulagnier (1989). Ainsi l'adolescent va reprendre des restes d'anciens vécus traumatiques, cette part d'insensé ayant sidérée sa psyché afin de tenter de se l'approprier.

\section{Le phénomène d'Après coup}

Les traces de l'impact traumatique s'observent donc, dans l'après coup. Elles se remobilisent nous explique Freud (1896) car elles traduisent que quelque chose n'a pu pleinement s'intégrer dans un contexte significatif au moment où cela a été vécu. Ces traces de scène ou vécu traumatique nous l'avons dit, se remobilisent à l'adolescence sous le joug de la pulsion pubertaire qui constitue un véhicule pour les transporter. Ainsi, les traces de ce qui n'a pu être assimilé, s'actualisent à l'aide d'une remise en scène pour être signifié et intégré. L'impact traumatique rend compte de la manière dont une situation à forte potentialité d'excitation, a effracté la psyché de l'enfant et aussi, témoigne de la façon dont l'enfant s'est retrouvé anéanti dans sa capacité à traiter et à donner sens à l'excitation qu'il a ressentie. Autrement dit, lorsqu'un environnement génère une excitation inintégrable, envahissante, de part sa violence, l'enfant absorbe la scène dans laquelle il est pris, quelque en soit sa nature qu'il soit témoin d'une scène ou impliqué directement. Cette absorption provoque un effet de collage psychique. Pour comprendre ce phénomène nous avons proposé la métaphore de la toile d'araignée (Blanquet, 2008).
Le modèle de la toile d'araignée:

Ce qui a fait traumatisme colle à la psyché de l'enfant comme s'il était prisonnier d'une scène, d'une toile. La scène comme la toile tissée, constitue un piège qui colle et enferme celui qui est piégé vers une problématique de destin qui reprend un vécu ancien de mort psychique. Cette métaphore de la toile d'araignée rend compte d'une part, des effets du trauma et d'autre part, des tentatives de solutions post traumatiques du sujet. Nous présentons trois composantes de ce modèle.

\section{Une construction paradoxale}

A l'adolescence, le sujet peut agir et provoquer, malgré lui ce qu'il redoute le plus car il est coincé dans une scène ayant eu un impact traumatique. Cette scène lui fait vivre une situation ambiguë, d'indécidable Duez (2002), qui brouille les places et les repères.

L'enfant ou l'adolescent, ne peut différencier: (1) ce qui a eu lieu et ce qui n'a pas eu lieu; (2) ce qui vient de lui et ce qui vient de l'autre ou des autres (s'il y a plusieurs interlocuteurs); (3) ce qu'il a vécu et ce qui appartient au vécu de l'autre ou des autres; (4) ce qui appartient au passé et au présent.

L'adolescent ne peut différencier la situation présente des situations passées car elles sont enchevêtrées. Il s'agit d'un véritable collapsus topique Janin (1996). Aussi lorsque le sujet tente de mettre en récit une scène actualisée, le psychologue ne sait plus de quelle scène il parle, tant ce qui s'est passé s'est mêlé au présent. L'adolescent se trouve dans un brouillage sensoriel et ne peut identifier ses ressentis qui resurgissent sur fond d'angoisse ou de vécu de mort psychique.

\section{Un vécu d'effraction psychique}

Ce modèle de traumatisme de type toile d'araignée, met l'accent sur une effraction du noyau central de la psyché dont les fragments ont volé en éclat. L'enfant est resté en suspension, figé hors du temps, englué dans la toile du traumatisme et en proie à des angoisses de mort psychique. L'adolescent peut de cette manière, vivre à nouveau des situations dans lesquelles il est en position de victime afin de juguler l'angoisse d'avoir servi de nourriture à un prédateur. Cela lui permet également de s'appuyer sur une nouvelle représentation susceptible de rassembler un reste de vécu traumatique précédant .

\section{Ce fonctionnement qu'illuste la toile d'araignée est de construction auto}

L'adolescent essaie dans l'après coup de reconstruire, à partir du modèle de l'effraction, l'histoire des traumatismes antérieurs. Ce modèle met en relief cette qualité particulière d'engluement. Il traduit toute la difficulté de décollement que le sujet rencontre, contraint à reprendre sur le même mode les effets de la survenue du traumatisme. Nous rapellons 
que la scène dans laquelle il fut englué enfant, fonctionne de manière ambiguë, paradoxale, et effractive. Et qu'il se trouve face à la nécessité de s'en construire une représentation.

Enfant, il a vécu une attaque de plein fouet dont les effets collent à sa peau psychique. C'est pourquoi il tente à l'adolescence de donner une figuration à son traumatisme en s'en construisant une représentation.

Après avoir décrit les effets traumatiques à l'aide de la métaphore de la toile d'araigneé, nous allons développer la notion de travail du traumatique.

\section{Le travail du traumatique:}

Ce travail rend compte de ce mouvement de reprise et s'observe de manière précise à l'adolescence. De façon générale, le travail du traumatique consiste pour un sujet à re-créer une scène, à partir de laquelle les traces de vécus traumatiques pourront se reprendre. La saisie de ces enjeux nous permettent d'expliciter la façon dont fonctionne le travail du traumatique. Il constitue l'arrière fond à partir duquel se déplie la nouvelle scène dont le but est de faire advenir à la conscience ce que cachent certaines traces traumatiques. A l'adolescence, la violence exercée par la puberté à l'encontre du corps ravive la dimension traumatique et accompagne cette mise en travail. C'est pour cela que nous observons plus facilement l'effet d'après coup car l'impulsion du pubertaire mobilise ce qui est resté en attente dans le psychisme pendant l'enfance. Le travail du traumatique fonctionne grâce à un processus que nous avons décrit: le processus de répétition-création Blanquet (2008). Ce processus est essentiel. Il est capital qu'il soit perçu dans sa double face.

\section{La répétition}

Le processus de répétition bien souvent décourage les professionnels, car ils observent que se répètent fréquemment, en apparence les mêmes situations surtout chez les adultes. En se décalant du piège du fonctionnement d'apparence chronique ou de type exclusivement décharge, nous pouvons interroger la dimension latente qui conduit le sujet à se retrouver régulièrement dans les mêmes situations.

Pourquoi l'adolescent ou même le sujet dit adulte, répètent t'ils ce qui semblent les avoir fait souffrir ? Ou pourquoi s'infligent t'ils ce qu'ils ont subi passivement ou l'infligent t'ils à un autre?

Nous proposons de considérer que ce processus de répétition sert au travail du traumatique de plusieurs façons. Tout d'abord le processus de répétition permet d'user les traces Roussillon (1995), qui sont restées encore active d'un vécu traumatique. Il s'agit là, de répéter pour user les traces afin qu'elles soient moins vivaces. D'autre part, un sujet agi en répétant ce qu'il a subi sur le principe du processus de retournement Roussillon (1991).

Ce processus permet de lutter face au retour d'un événement qui lui a fait éprouvé, un vécu de détresse sans secours ni recours et bien souvent assorti d'un vécu de mort psychique. Le sujet tente d'éviter le retour de l'événement désorganisateur en le provoquant afin de le maitriser pour tenter de s'approprier ce qui dans la première scène vécue, l'a désorganisé. Le sujet peut ainsi anticiper ce qui va se passer. Il peut même déclencher chez l'autre un comportement pour tenter d'en maitriser les effets par anticipation. Il s'agit là de faire vivre activement ce qui a été subi dans le but pour se l'approprier et éviter à nouveau la survenue d'une désorganisation psychique.

Ce processus de répétition est intéressant aussi car il permet au sujet de récréer une scène qui essaie d'intégrer les restes traumatiques et de les relier entre eux. C'est ce que Marty (2002) appelle le travail du lien. Le sujet tente de ré-éprouver les sensations restées en panne de symbolisation. Il s'agit là, de revivre des sensations fortes, afin de lier entre elles, les impressions sensorielles et produire ainsi une zone de perception permanente. Cette zone de permanence ainsi créée, constitue un moyen radical de lutte face à l'angoisse de mort.

Le travail du traumatique a pour objectif premier de faire advenir quelque chose qui n'était pas possible en temps précoce. Il met en mouvements les traces traumatiques pour permettre à un sujet de fabriquer des images, de sentir, de ressentir et de trouver un chemin de restitution selon Maldavsky (1998). Le sujet essaie de contenir un ensemble de vécu qui cherche à se frayer un chemin et à se signifier dans le psychisme.Il s'agit là d'une manière de créer à l'extérieur une scène afin de loger ce qui travaille et agite le sujet de l'intérieur.

Face aux traitements des traces de vécus traumatique, le processus de répétition est nécessaire au sujet. Il fonctionne de concert avec que nous avons nommé le processus de création.

\section{La création}

Il s'agit pour un sujet de répéter au sens de repasser par un mouvement de reprise en introduisant une légère différence. Pour tous sujet, l'accès direct au traumatisme est impossible. Il est donc nécessaire de recréer en répétant une à des scènes à potentialité traumatique dans des conditions plus favorables. La répétition à l'identique est peut efficace. En revanche si le sujet répète en introduisant une légère différence, en faisant varier les données de la survenue de l'expérience, il devient créatif. La capacité créatrice du sujet lui permet d'ouvrir un espace susceptible de l'aider dans ce travail de construction.

\section{Ilustration clinique}

Nous proposons d'illustrer l'importance de ce processus répétition/création en nous appuyant sur une vignette clinique. Il s'agit de la situation d'une jeune adolescente Liriopé âgée de dix huit dont le cas détaillé est publié dans la revue Adolescence, Blanquet (2010).

Je rencontre Liriopé à ses dix huit ans sur les conseils de son éducateur. Quelques mois plus tôt, Liriopé fut victime d'une agression sexuelle. La scène de l'agression nous montrera la manière dont le processus répétition /création 
est à l'œuvre dans le travail du traumatique. Son agresseur vivait dans la même résidence qu'elle Elle lui aurait sa porte. Il l'aurait menacé avec une arme afin d'avoir des relations sexuelles. Lorsqu'au petit matin, il sort de chez elle, elle croise le regard du veilleur de nuit, puis se rend au commissariat porter plainte. Suite à la plainte qu'elle a déposée, son agresseur est en prison. Elle vient me voir pour selon ces propos, 'que je la prépare à affronter le procés'. Lors de cette première séance, elle fait le récit pathétique, de la violence dont elle a été victime. Pour Liriopé 'les choses lui tombent dessus et justice doit être faite'.

Durant cette première séance, les mots se déroulent, les époques s'emmêlent. Le récit de la scène de l'agression laisse entrevoir d'anciens restes traumatiques agglutinés à celui là. Ces restes émergent et se télescopent à des images parentales, lorsqu'elle évoque la situation subie. Pendant l'entretien, elle parle incidemment, du départ de sa mère et aurait aimé que son père soit là pour la protéger.

Au bout d'un an de prise en charge psychothérapique, les éléments intriqués et condensés par la scène de l'agression semblent se dénouer. Liriopé parle de ses rêves à partir desquels elle essaie de mettre en forme ces éprouvés au moment de l'agression. Elle rêve de quatre chatons se trouvant enfermés dans un sac à l'arrière d'une voiture. Puis se souvient d'une image flash qu'elle a vu pendant l'agression. Il s'agit d'une voiture bleue. Elle se rappelle également qu'au moment de l'agression elle voulait crier «papa » mais aucun son n'est sorti de sa bouche.

Liriopé a connu précocement un contexte de forte mésentente conjugale. Elle est la dernière enfant d'une fratrie. Sa venue au monde même était censée réconcilier ses parents, ce qui ne fut pas le cas. A contrario, sa mère un jour l'amena ainsi que ses frères et sœurs dans la famille de son père et ne les revit plus pendant de nombreuses années. Liriopé était enfant mais se souvient d'avoir été installée à l'arrière d'une voiture. Elle ne remarqua la couleur bleue qu'au moment où elle la vit disparaître. Elle grandit avec ses frères et sœurs principalement chez son père.

Elle se souvient également d'une autre scène qui faisait d'elle la victime de son frère. Une fois son père est entré dans la chambre et a vu son fils abusait d'elle. Il a refermé la porte et n'en a rien dit. Ce silence de son père, sa non intervention et aussi le départ de sa mère rendent compte de la vivacité des traces de vécus traumatiques qui n'ont pu être mises en mots et en sens. A l'adolescence pourtant, Liriopé tenta de porter plainte contre son frère mais cela n'aboutit pas car elle se rétracta.

Probablement aussi qu'elle essaya par le biais de relation sexuelle avec des garçons violents de reprendre les nœuds de son histoire. Ceci-dit, Liriopé a vécu de nombreux traumatismes liés à des vécus de détresse, de lâchage précoce, à de nombreuses expériences d'absence de secours maternel et paternel, et aussi à un climat environnemental chargée d'excitation non régulée.

A travers cet exemple nous observons la manière dont le processus de répétition/création est à l'œuvre et au service du travail du traumatique Potamianou (2001). Liriopé montre dans cette situation comment les traces des événements antérieurs sont conservés à l'état de traces mnésiques. Ces traces tentent de s'actualiser sur une scène qui présentent des analogies avec des situations d'effraction antérieures. Se revit alors des éprouvés qui resurgissent sous forme perceptive, comme des retours d'éléments clivés Roussillon (1999). Ces retours rendent compte d'une non-réponse de la part de l'environnement, d'une souffrance n'ayant pas fait l'objet d'une expérience partageable. L'absence de traces représentatives, reste en mouvement comme une lettre sans destinataire, qui se fait l'écho d'une souffrance. Cependant, l'exemple de Liriopé s'appuie aussi sur une création. Elle parvient à localiser la figure de l'agresseur à l'extérieur de sa famille pour tenter de sortir de l'ambiguïté Bleger (1967), ou de l'indécidabilité Duez (2002). Grâce à cet écart introduit entre la scène de l'agression, et les indices qui permettent de faire le lien avec les anciens traumatismes, Liriopé ouvre un espace qui facilite l'accès aux images traumatiques ainsi rendues figurables Houssier (1999).

Le monde social reconnaît l'agression. Liriopé est reconnue victime. Elle montre à qui le souhaite les papiers du tribunal attestant de la culpabilité et perversité de son agresseur c'est écrit clame t'elle. Elle s'accroche à cette attestation du tribunal jusqu'à dormir avec elle car cela constitue une preuve irréfutable que cela vient de l'autre, et que c'est l'autre. La convocation de la loi permet de légitimer ce qui s'est passé, et lui confère une reconnaissance de ce qui a été subi dans cette scène là et toutes celles que la scène de la violence, de l'effraction condensent. Liriopé peut ainsi localiser la figure du fauteur d'excitation selon Potamianou (2001).

\section{Conclusion}

Liriopé tente d'advenir à partir de ce processus répétition/ création pour essayer de se souvenir et de traiter les restes traumatiques dans sa psyché. Sa capacité créatrice, c'est à dire la construction après coup du traumatisme qu'elle effectue, s'apparente à une mise en lien de traces. Le travail psychothérapique dans lequel elle s'est engagée lui a permis de s'approprier et transformer en représentation les expériences ayant excédé ses capacités d'intégration. Il fallut à Liriopé, l'extériorisation de situations dramatiques chargées des traces d'anciens vécus traumatiques, pour ré-éprouver l'affect de mort psychique et le localiser psychiquement.

L'histoire de Liriopé illustre un phénomène de reprise des expériences traumatiques de l'enfance, pour tenter à l'adolescence, de se construire une identité.

\section{Références}

Aulagnier, P. (1989). Se construire un passé, in le narcissisme à 1'adolescence, Colloque de Monaco, Journal de la psychanalyse de l'enfant, 7, 191-221.

Blanquet, B. (2008). L'ordalie du contact: un analyseur de la scène ordalique. Thèse de $3^{\circ}$ cycle, Université Lumière Lyon2.

Blanquet, B. (2010). Frôler la mort à l>adolescence: un danger nécessaire. Revue Adolescence, 28, 123-131.

Bleger, J. (1967). Symbiose et ambiguïté, coll. Le fil rouge. Paris: Les Presses Universitaires de France- PUF.

Duez, B. (2002). L'indécidabilité: un modèle générique du traumatisme, Perspectives Psy, 41(2), 113-118. 
Ferenczi, S. (1932). Réflexion sur le traumatisme, in Euvres complètes IV, Paris: Payot. pp139-147.

Freud, S. (1896). Lettres à Wilhelm Fliesb 1887-1904. Paris: Les Presses Universitaires de France- PUF.

Gutton, P. (1991). Le pubertaire, Paris: Les Presses Universitaires de France- PUF.

Houssier, F. (1999). Le passage à l'acte: réflexion sur un concept, in Psychologie clinique, l'Harmattan, 125-134.

Janin, C. (1996). Figures et destin du traumatisme. Paris: Les Presses Universitaires de France- PUF.

Maldavsky, D. (1998). Les névroses toxiques et traumatiques. Cauchemard en veille. Paris: L'Harmattan.

Marty, F. (2002). Le lien et quelque-unes de ses figures. Rouen: Publcations des Universités de Rouen et du Havre - PURH.

Potamianou, A. (2001). Le traumatique, Répétition et élaboration. Paris: Dunod.
Roussillon, R. (1991). Paradoxes et situations limites de la psychanalyse. Paris: Les Presses Universitaires de FrancePUF.

Roussillon, R. (1995). Logique et archéologique du cadre psychanalytique. Paris: Les Presses Universitaires de FrancePUF.

Roussillon, R. (1999). Agonie, clivage et symbolisation. Paris: Les Presses Universitaires de France- PUF.

Recebido em 01.05.2009

Primeira decisão editorial em 03.05.2010

Versão final em 03.11.2010

Aceito em 04.11.2010 